\title{
Modélisation de l'irrigation en goutte à goutte enterré du palmier dattier sous les conditions oasiennes
}

\author{
Rqia Bourziza ${ }^{1, *}$, Ali Hammani ${ }^{1}$, Jean Claude Mailhol ${ }^{2}$, Ahmed Bouaziz ${ }^{1}$ et Marcel Kuper ${ }^{1,3}$ \\ ${ }^{1}$ IAV Hassan II, Madinat Al Irfane, BP 6202, 10101 Rabat, Maroc \\ 2 Irstea UMR G-eau, 361, rue J.F.-Breton, BP 5095, 34196 Montpellier cedex 05, France \\ ${ }^{3}$ Cirad UMR G-eau TA C-90/15 L'Hortus, 73, rue J.F.-Breton, 34398 Montpellier cedex 5, France
}

\begin{abstract}
Résumé - En raison de sa bonne efficience potentielle, l'irrigation en goutte à goutte enterré (GGE) a été récemment introduite au Maroc. Ce document discute son utilisation pour le palmier dattier dans l'oasis du Tafilalet (Sud-Est du Maroc), où une conception et une gestion appropriée de ce système doivent être proposées aux agriculteurs. L'objectif de l'article est d'évaluer l'effet de la variation de la profondeur d'installation du système GGE entourant le palmier dattier sur la distribution de l'humidité dans le sol. Pour atteindre cet objectif, un modèle numérique simulant le transfert multidirectionnel de l'eau du sol a été utilisé. Les résultats de la simulation à l'aide d'un modèle bidimensionnel axisymétrique ont été comparés à des mesures effectuées sur un sol limoneux, dans la parcelle d'un agriculteur où le GGE a été installé à différentes profondeurs. L'étude montre la pertinence du modèle pour simuler l'infiltration autour d'une rampe enterrée. L'humidité du sol est plus stable avec le GGE à $35 \mathrm{~cm}$ de profondeur en comparaison avec une installation à $15 \mathrm{~cm}$ ou $25 \mathrm{~cm}$ de profondeur. Il y a aussi une augmentation de la teneur en eau volumique sous la surface du sol pour la profondeur de $35 \mathrm{~cm}$, par rapport aux profondeurs de $15 \mathrm{~cm}$ et $25 \mathrm{~cm}$, du fait de la diminution de l'évaporation.
\end{abstract}

Mots clés : oasis / palmier dattier / goutte à goutte enterré / modèle bidimensionnel axisymétrique / infiltration

Abstract - Modeling subsurface drip irrigation for date palm under oasis conditions. Due to its high potential efficiency, subsurface drip irrigation (SDI) was recently introduced in Morocco. This paper deals with its application to the date palm in the Tafilalet oasis (south-east of Morocco), where appropriate design and management of this system have to be proposed to farmers. The objective of this paper is to assess how the variation of the installation depth of an SDI system surrounding a date palm tree can affect the distribution of soil moisture. A numerical model simulating the multidirectional soil water transfer was used to evaluate the distribution of water around the dripper line in the local silty soil conditions. The simulation results using an axisymmetric two-dimensional model were compared to field measurements carried out in a farmer's plot where SDI was installed at different depths. The study shows the suitability of the model to simulate infiltration around a dripper line during irrigation. Soil moisture is steadier with SDI at $35 \mathrm{~cm}$ depth than with SDI at $15 \mathrm{~cm}$ and $25 \mathrm{~cm}$. There is an increase in volumetric soil water content below the soil surface for a $35 \mathrm{~cm}$ depth, compared to $15 \mathrm{~cm}$ and $25 \mathrm{~cm}$, due to less evaporation.

Keywords: oasis / date palm / subsurface drip irrigation / axisymmetric two-dimensional model / infiltration

\section{Introduction}

Dans les oasis, les ressources en eau sont de plus en plus rares et souvent de mauvaise qualité. L'utilisation de techniques d'irrigation innovantes et efficientes, pour économiser l'eau, est une option intéressante pouvant contribuer à la pérennité des oasis. La technique de l'irrigation en goutte à goutte enterré (GGE) pourrait être une alternative viable puisqu'elle économise l'eau en situation de forte demande

\footnotetext{
* Auteur de correspondance : rqia.bourziza@gmail.com
}

évaporative de surface. La technique du GGE se distingue du système classique de goutte à goutte posé à même le sol, du fait que le système est installé de manière pérenne à plusieurs dizaines de centimètre dans le sol, ne perturbant ainsi plus le travail du sol (désherbage, labour), contrairement au goutte à goutte de surface classique. L'irrigation en GGE peut également fonctionner avec des eaux usées épurées, ce qui rendrait l'utilisation de ce type de ressource plus acceptable pour les agriculteurs. Le système de GGE présente en outre l'avantage d'apporter l'eau près des racines, améliorant encore les performances de l'irrigation; la raison avancée est qu'elle empêche ou réduit considérablement les pertes par évaporation 
directe, le ruissellement et la percolation profonde (Hanson et May, 2007 ; Safi et al., 2007). L'efficience d'utilisation de l'eau pour un système GGE bien conçu peut être supérieure à $95 \%$ (Payero et al., 2005). Toutefois, le GGE n'échappe pas aux risques de colmatage. Aussi, les consignes de filtration et de maintenance doivent-elles être bien respectées ; il est notamment recommandé de se doter de moyens de contrôle des pressions dans les conduites pour détecter un éventuel colmatage. En outre, les matériaux utilisés ne sont pas biodégradables, ce qui, pour respecter les contraintes environnementales, nécessitera, en fin de vie des tuyaux, une récupération plus ou moins coûteuse des dispositifs enterrés (Mailhol et al., 2013). L'inconvénient majeur du GGE est le coût élevé de l'installation en comparaison avec d'autres systèmes d'irrigation. Cependant, dans la mesure où la durée de vie de 15 ans annoncée par les constructeurs peut être effectivement atteinte, d'un point de vue économique le GGE surpasserait la technique du goutte à goutte de surface (GGS), dans la mesure où il permet une amélioration des rendements des cultures observée sous différentes conditions pédoclimatiques à travers le monde (Camp, 1998).

L'eau délivrée par un goutteur enterré mouille un volume de sol, qui a la forme d'un ellipsoïde tronqué le volume de cet ellipsoïde tronqué est caractérisé par des valeurs spécifiques de sa profondeur, $z$, et de sa largeur maximale, $d$, qui sont inversement liées (Zur, 1996). La connaissance de la dynamique de l'eau dans le volume de sol entourant l'émetteur est une condition préalable à la conception de systèmes d'irrigation, ainsi que pour la gestion de l'eau et les intrants (Akbar et al., 1996; Zur, 1996). Les modèles d'humectation peuvent être obtenus soit par des expériences au champ, soit par simulation. La forme d'humectation du sol soumis à une irrigation souterraine a été simulée par de nombreux auteurs. Lazarovitch et al. (2007) ont simulé la forme d'humectation pour un système d'irrigation GGE en utilisant la technique d'analyse dite des moments. Elle consiste à calculer l'avancement du front d'humectation, pour le premier moment (le temps zéro qui représente le moment de l'irrigation) et les moments qui suivent, et à définir un ellipsoïde qui décrit la forme et l'orientation moyenne du bulbe pour chaque période d'observation. Elmaloglou et Diamantopoulos (2009) ont présenté un modèle pour simuler la dynamique de l'eau du sol en irrigation au GGE en tenant compte de l'évaporation et de l'extraction racinaire. Diamantopoulos et Elmaloglou (2012) ont également montré que lorsque la profondeur de placement des rampes augmente, le mouvement horizontal de l'eau diminue à proximité de la surface. Mailhol et al. (2001) ont réalisé une expérimentation sur un sol limoneux, pour la culture du maïs, afin d'identifier la meilleure stratégie d'irrigation et de fertigation, en irrigation gravitaire et par aspersion. Ils ont observé que le modèle Hydrus-2D (Šimůnek et al., 1999) pouvait prédire la distribution d'azote pour les deux types d'arrosage. Kandelous et Šimůnek (2010) ont aussi testé le modèle Hydrus-2D en comparant les conditions au laboratoire et les conditions au champ dans le cas du GGE; la correspondance entre les simulations et les observations était bonne. Assouline (2002) et Abbasi et al. (2003a, 2003b) ont aussi montré que les simulations Hydrus-2D de la teneur en eau du sol et des distributions de soluté étaient raisonnablement proches des valeurs mesurées. Selon les études de Skaggs et al. (2004), Provenzano (2007) et Kandelous et Šimůnek (2010), Hydrus-2D peut décrire de manière satisfaisante la répartition de l'eau dans le cas de l'irrigation en GGE, dans les conditions du laboratoire ou dans celle du champ. Ajdary et al. (2005) ont calibré Hydrus-2D avec les données observées pour une culture d'oignon dans un sol sablo-limono-argileux irrigué en goutte à goutte.

Comme dans de nombreuses régions du monde, l'interaction entre le climat, les sols et la production agricole dans les oasis présente des combinaisons uniques qui nécessitent des recherches locales pour mettre au point les systèmes de culture. L'objectif de cet article est d'évaluer dans quelle mesure la variation de la profondeur d'installation du système GGE entourant les jeunes palmiers dattiers peut affecter la distribution de l'humidité du sol.

\section{Matériels et méthodes}

\subsection{Expérimentation et mesures}

Le site expérimental est situé dans l'oasis du Tafilalet, au Sud-Est du Maroc ( $31^{\circ} 22^{\prime} 38^{\prime \prime} \mathrm{N}, 4^{\circ} 17^{\prime} 7^{\prime \prime}$ O). Une station météorologique entièrement équipée est installée sur le site pour avoir les données climatiques telles que la température, l'humidité de l'air, la pluviométrie et l'évapotranspiration potentielle. Les expériences de terrain ont été réalisées sur un sol limoneux contenant en moyenne $52 \%$ de limon, $27 \%$ de sable et $21 \%$ d'argile dans un profil de $1 \mathrm{~m}$ de profondeur, avec une densité apparente moyenne de $1,76 \mathrm{~g} . \mathrm{cm}^{-3}$. Un mélange de vitro-plants et de rejets ont été plantés en avril 2012 avec un espacement de $7 \mathrm{~m} \times 7 \mathrm{~m}$ (112 plants par hectare). Un apport de sable, de fumier et d'engrais de fond $\left(0,3 \mathrm{~m}^{3} \mathrm{de}\right.$ sable $+5 \mathrm{~kg}$ de fumier $+2 \mathrm{~kg}$ d'engrais de fond) a été ajouté pour remplir les trois quarts de chacune des fosses de plantation pour réduire la densité du sol et l'enrichir en matière organique. La moyenne de la réserve utile (RU) du sol étudié est de $186 \mathrm{~mm}$ pour $1 \mathrm{~m}$ de sol modifié, ce qui représente en moyenne une réserve facilement utilisable (RFU) pour les plantes de $75 \mathrm{~mm}$ à une profondeur de $60 \mathrm{~cm}$. L'humidité à la capacité au champ de ce sol est de $23 \%$ et son humidité au point de flétrissement est de $12 \%$.

Deux expériences ont été menées pour comparer les méthodes d'irrigation. La première expérience concerne des rampes circulaires enfouies à différentes profondeurs $(15,25$ et $35 \mathrm{~cm}$ sous la surface du sol). Le débit moyen des goutteurs était de $2 \mathrm{~L} . \mathrm{h}^{-1}$ à une pression de $1 \mathrm{bar}$, avec un espacement entre les goutteurs de $0,4 \mathrm{~m}$. La seconde expérience a été réalisée avec des rampes circulaires installées sur la surface du sol avec des goutteurs de $2 \mathrm{~L} . \mathrm{h}^{-1}$ et un espacement entre goutteurs de $0,4 \mathrm{~m}$. Pour les deux systèmes, nous avons 24 goutteurs autour de chaque palmier dattier. Pendant toute la période de l'expérimentation, les doses d'irrigation ont été les mêmes pour le GGE et pour l'irrigation au GGS.

Les appareils de mesure de l'état hydrique du sol ont été installés pour chaque expérience (Fig. 1). La sonde Delta-T (PR2) a été étalonnée avec des mesures gravimétriques sur des échantillons de sol prélevés dans trois placettes du site expérimental (sol saturé, sol à la capacité au champ et sol sec), pour calculer l'humidité volumétrique du sol. Pour chaque profondeur de pose de rampes, nous avons installé trois sondes Delta-T. Les sondes Delta-T de type PR2 mesurent l'humidité du sol sur six profondeurs allant de $10 \mathrm{~cm}$ à $100 \mathrm{~cm}$. Elles nécessitent un trou d'installation d'un peu plus de $27 \mathrm{~mm}$ de 


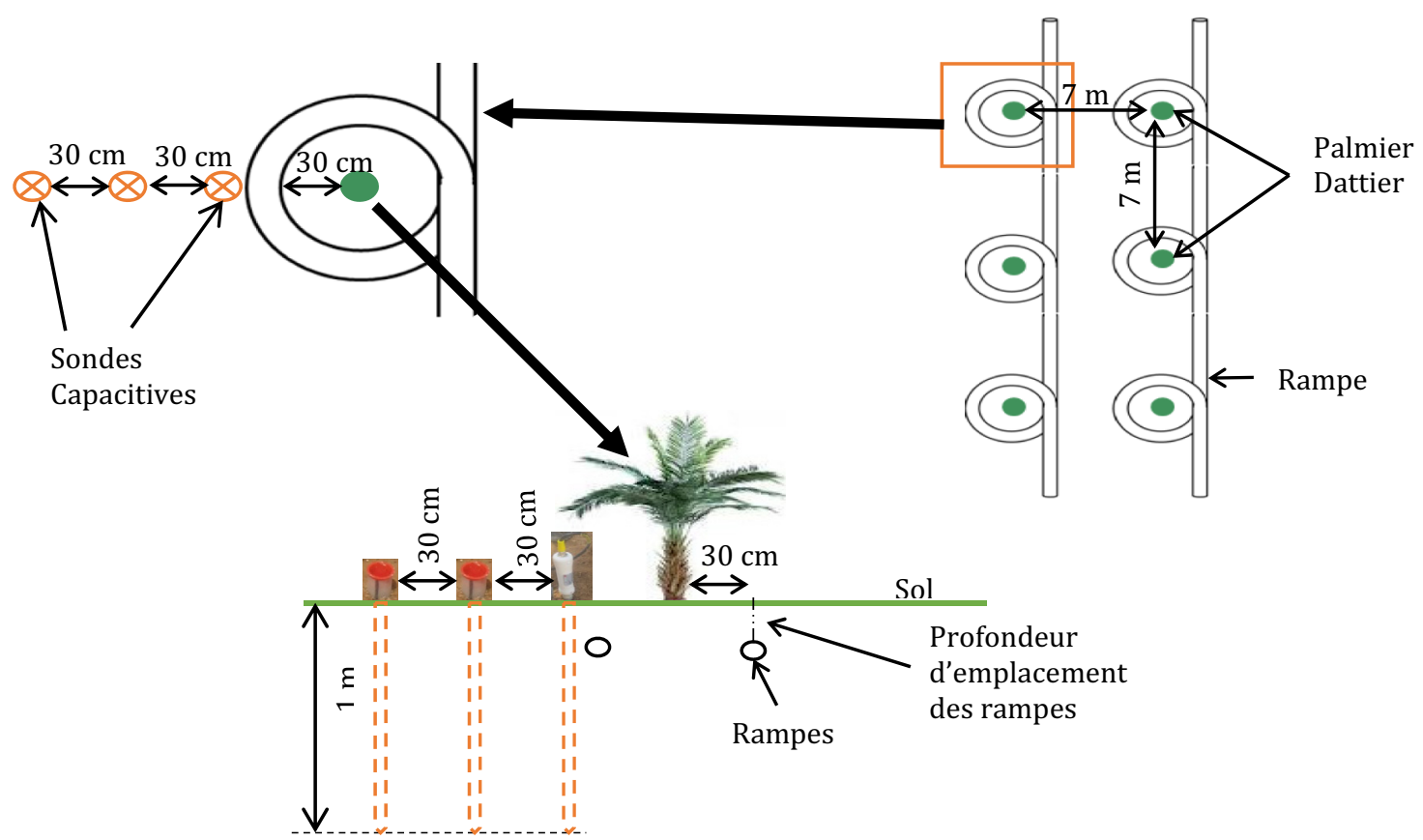

Fig. 1. Protocole expérimental utilisé pour l'installation des rampes d'irrigation et des sondes capacitives.

Fig. 1. Experimental protocol used for irrigation laterals and capacitive probes installation.

diamètre, ce qui permet une installation facile en minimisant la perturbation du sol. Ces sondes fonctionnent d'après un principe en relation avec la variation de la constante diélectrique. Les relevés sont effectués grâce à un boîtier mobile qui permet la lecture de plusieurs sondes. Ceci est possible grâce à un signal généré, s'appliquant sur une ligne de transmission qui pénètre dans le sol à travers une paire d'anneaux en acier inox. L'eau contenue dans le sol autour de l'anneau détermine la quantité du signal émis qui rayonne dans le sol et la quantité de signal qui sera réfléchie à l'interface entre la ligne de transmission et l'anneau de mesure. La partie réfléchie du signal se combine avec le signal émis pour donner une onde entretenue le long de la ligne de transmission et l'amplitude de cette onde est directement fonction de la teneur en eau du sol. Ce dispositif a été utilisé afin de suivre la variation de la teneur en eau du sol latéralement et en profondeur, pour caractériser le bulbe d'humectation et pouvoir calibrer et valider le modèle Hydrus-2D.

\subsection{Modèle numérique Hydrus 2D}

Avec l'hypothèse d'un sol homogène, isotrope, variablement saturé et initialement uniforme, l'écoulement est considéré comme axisymétrique, c'est-à-dire symétrique par rapport à l'axe vertical passant par l'arbre (cas d'une infiltration à partir d'une rampe entourant l'arbre, où les goutteurs sont suffisamment rapprochés). L'équation de Richards (Hillel, 1980) régit le mouvement de l'eau dans un sol homogène et isotrope ; cette équation est détaillée en système de coordonnées cylindriques :

$$
\frac{\partial \theta}{\partial t}=\frac{1}{r} \frac{\partial}{\partial r}\left[r K(h) \frac{\partial h}{\partial r}\right]+\frac{\partial}{\partial z}\left[K(h) \frac{\partial \boldsymbol{h}}{\partial z}+K(\boldsymbol{h})\right]+R_{\mathrm{e}}
$$

où $\theta$ est la teneur en eau volumique $\left[\mathrm{L}^{3} \mathrm{~L}^{-3}\right], h$ est le potentiel matriciel $[L], t$ est le temps $[T], r$ est la coordonnée radiale $[L]$, $z$ est la coordonnée verticale $[L], K$ est la conductivité hydraulique [ $\mathrm{LT}^{-1}$ ] et $R_{\mathrm{e}}$ décrit la quantité d'eau extraite par les racines par unité de volume de sol et unité de temps (taux d'extraction racinaire) $\left[\mathrm{L}^{3} \mathrm{~T}^{-1} \mathrm{~L}^{-3}\right]$.

La résolution de cette équation en tenant compte des conditions aux limites et des conditions initiales, permet de déterminer en tout point du milieu poreux et en tout temps le potentiel matriciel $h$ et d'en déduire la vitesse de Darcy par la loi de Darcy. L'équation de Richards est une équation nonlinéaire dont la résolution nécessite la connaissance de deux fonctions décrivant les propriétés hydrodynamiques du sol: la courbe de rétention d'eau $\theta(h)$ et la courbe de conductivité hydraulique $K(h)$. Les propriétés hydrodynamiques du sol ont été modélisées à l'aide des relations constitutives de van Genuchten-Mualem (Mualem, 1976 ; Van Genuchten, 1980), comme suit :

$$
\begin{gathered}
\theta(\boldsymbol{h})= \begin{cases}\theta_{r}+\frac{\theta_{s}-\theta_{r}}{\left(1+|\alpha h|^{n}\right)^{m}} & \boldsymbol{h} \geq \mathbf{0} \\
\theta_{s}\end{cases} \\
K(\boldsymbol{h})=K_{s} S_{e}^{l}\left[1-\left(1-S_{e}^{\frac{1}{m}}\right)^{m}\right]^{2},
\end{gathered}
$$

où $S_{e}=\frac{\theta-\theta_{r}}{\theta_{s}-\theta_{r}}, m=1-\frac{1}{n}, \theta_{\mathrm{s}}$ est la teneur en eau à saturation $\left[\mathrm{L}^{3} \mathrm{~L}^{-3}\right], \theta_{\mathrm{r}}$ est la teneur en eau résiduelle $\left[\mathrm{L}^{3} \mathrm{~L}^{-3}\right], K_{\mathrm{s}}$ est la conductivité hydraulique à saturation $\left[\mathrm{LT}^{-1}\right]$, et $\alpha\left[\mathrm{L}^{-1}\right]$ inverse de la pression d'entrée d'air, $n[-]$ indice de distribution de la taille des pores et $l[-]$ paramètre empirique généralement lié à la tortuosité des pores du sol $(l=0,5$ est la valeur par défaut largement utilisée dans la littérature). Les paramètres hydrauliques de Van Genuchten-Mualem du sol 
étudié, obtenus après calibration, sont donnés dans le Tableau 1.

HYDRUS-2D utilise la méthode des éléments finis pour résoudre l'équation régissant l'écoulement de l'eau. Pour les simulations d'irrigation souterraine, la solution est présentée dans un domaine rectangulaire, situé dans un plan vertical (Fig. 2). L'emplacement du cercle représentant le goutteur dépend de l'emplacement du goutteur dans le champ. Deux types de matériaux ont été utilisés pour la simulation du domaine étudié, puisque nous avons effectué un ajout de sable dans un volume d'un mètre cube de sol pendant la plantation des jeunes palmiers dattiers pour améliorer la densité et par conséquent la perméabilité du sol.

La hauteur du domaine est de $4 \mathrm{~m}$ puisque les racines du palmier dattier, selon l'étude faite par El Khoumsi et al. (2014), ne dépassent pas $4 \mathrm{~m}$ de profondeur dans cette région. La largeur du domaine est de 3,5 m puisque l'espacement entre les palmiers dattiers est de $7 \mathrm{~m}$. Le domaine est discrétisé en éléments finis selon un maillage régulier triangulaire. Pour les conditions aux limites (Fig. 2), une limite de flux nul a été utilisée sur les limites latérales verticales du profil du sol, parce que le mouvement sol-eau sera symétrique le long de ces frontières. Les conditions de flux volumique variable du goutteur ont été introduites pour tenir compte de la variabilité

Tableau 1. Paramètres hydrodynamiques du sol après calibration du modèle Hydrus-2D.

Table 1. Hydrodynamic parameters of soil after calibration of Hydrus-2D model.

\begin{tabular}{lllllll}
\hline Matériaux & $\begin{array}{l}\theta_{\mathrm{r}} \\
\left(\mathrm{m}^{3} \mathrm{~m}^{-3}\right)\end{array}$ & $\begin{array}{l}\theta_{\mathrm{s}} \\
\left(\mathrm{m}^{3} \mathrm{~m}^{-3}\right)\end{array}$ & $\begin{array}{l}\alpha \\
\left(\mathrm{m}^{-1}\right)\end{array}$ & $\begin{array}{l}n \\
(-)\end{array}$ & $\begin{array}{l}K_{\mathrm{s}} \\
\left(\mathrm{m} \mathrm{j}^{-1}\right)\end{array}$ & $\begin{array}{l}1 \\
(-)\end{array}$ \\
\hline 1 & 0,04 & 0,31 & 1,83 & 2,12 & 0,46 & 0,5 \\
2 & 0,05 & 0,34 & 2,43 & 1,93 & 0,96 & 0,5 \\
\hline
\end{tabular}

des doses apportées par irrigation. Le flux d'eau est obtenu en divisant le débit d'eau par la surface de l'émetteur lors des irrigations. Durant la période d'absence d'irrigation, le flux est maintenu à zéro. La limite inférieure a été définie par un gradient hydraulique vertical, simulant le drainage libre à partir d'un profil de sol relativement profond (Rassam et Littleboy, 2003). L'évaporation Ep (\% d'ETC) et la transpiration Tp (\% d'ETC) ont été données comme entrées dans le tableau des conditions aux limites de la période de simulation choisie (Patel et Rajput, 2008).

\section{Résultats et discussions}

\subsection{Validation}

La première étape est de valider les paramètres du modèle Hydrus-2D dans le cas de notre étude. La validation consiste en premier lieu à comparer les valeurs mesurées et calculées de la teneur en eau du sol et en seconde lieu à calculer les indices de performance statistique. Les teneurs en eau mesurées et simulées pour les trois profondeurs de pose $(15,25,35 \mathrm{~cm})$ sont présentées sur la Figure 3.

Dans l'ensemble, les valeurs de la teneur en eau simulées par le modèle Hydrus-2D sont en accord avec les valeurs mesurées, en dépit de quelques divergences. Malgré la complexité du scénario de simulation et les propriétés composites du sol liées à ses deux matériaux, la simulation par modèle aboutit à un accord plausible avec les données expérimentales. Le modèle a été soumis à plusieurs événements consécutifs d'irrigation, à une évapotranspiration variable et à un temps de distribution des racines statique pour une telle période de simulation (une des limites du modèle Hydrus-2D étant qu'il ne permet pas l'entrée d'un système racinaire dynamique). Parmi les indices statistiques généralement utilisés pour la validation du modèle, nous avons choisi la racine carrée de l'erreur quadratique moyenne (RMSE).
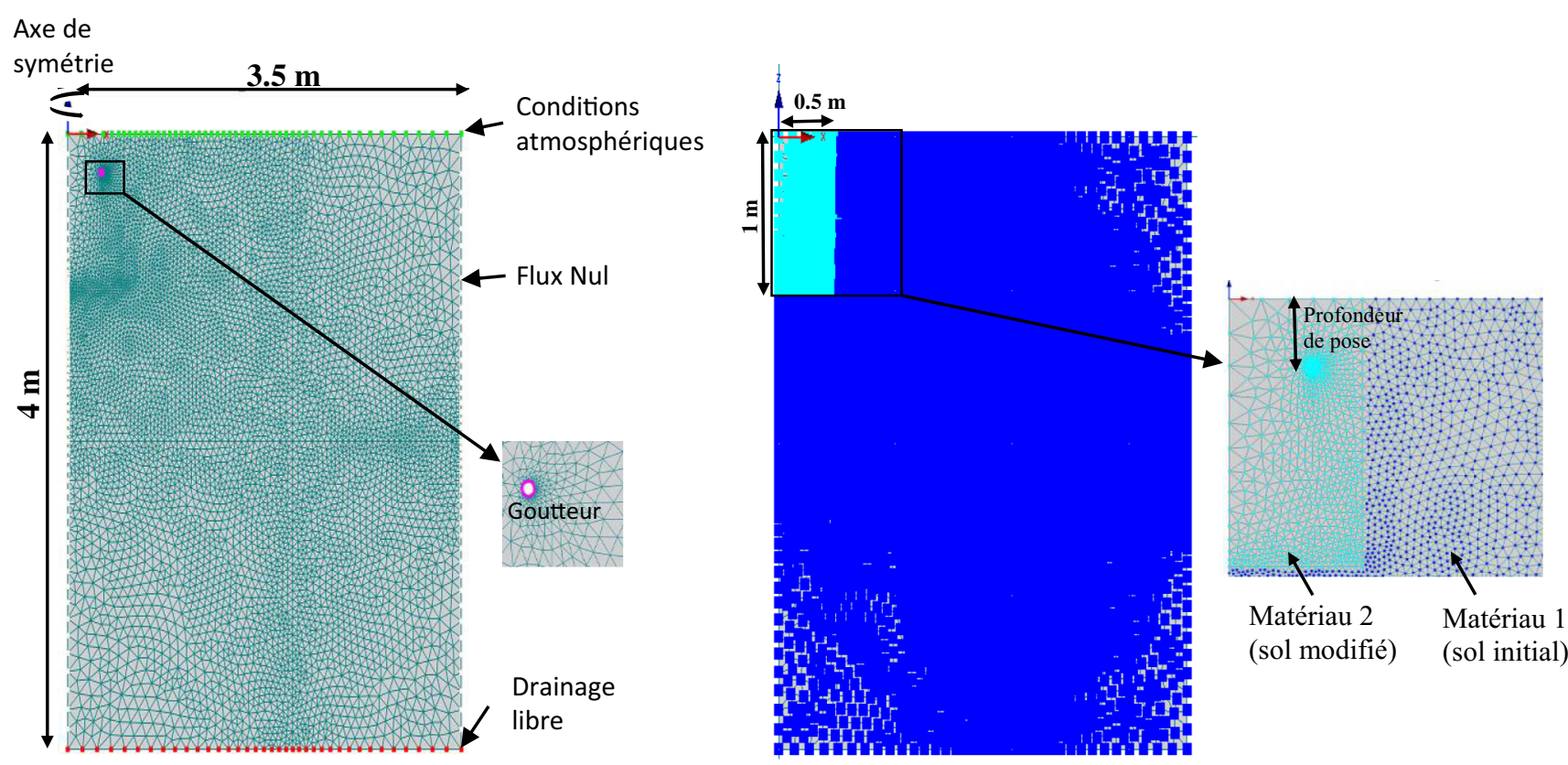

Fig. 2. Construction des conditions aux limites et des différents matériaux utilisés pour le modèle Hydrus-2D dans le cas du goutteur enterré. Fig. 2. Construction of boundary conditions and the different materials used for Hydrus-2D for a buried emitter. 


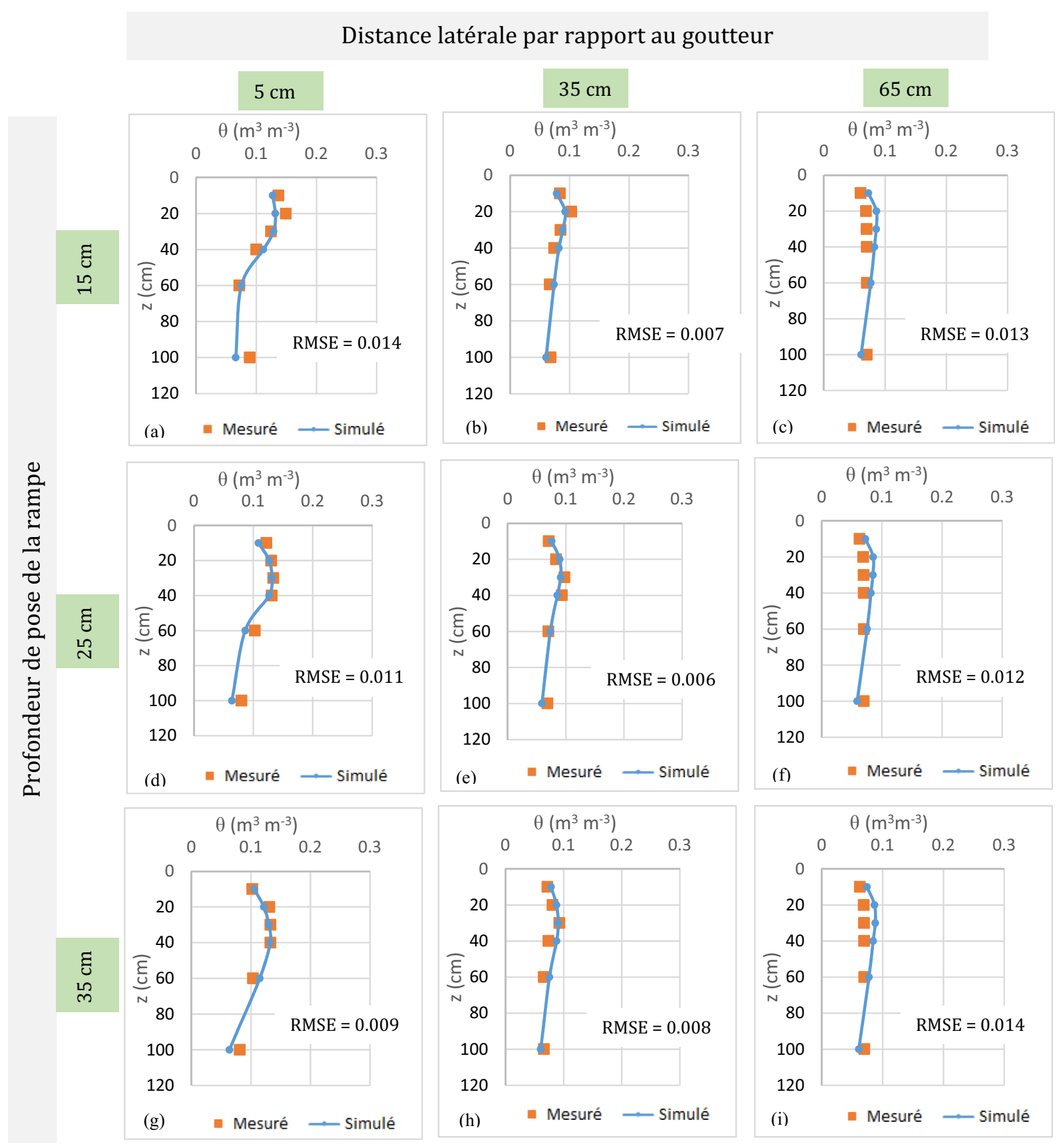

Fig. 3. (a) à (i) Teneurs en eau volumiques $(\theta)$ simulées et mesurées pour différentes positions latérales par rapport au goutteur respectivement à 5,35 et $65 \mathrm{~cm}$ et en fonction de la profondeur de placement des rampes (DOY 154).

Fig. 3. Simulated and measured volumetric water content $(\theta)$ from 5,35 and $65 \mathrm{~cm}$ of laterals positions relative to the emitter and depending on depth placement of laterals (DOY 154).

Les valeurs du RMSE oscillent entre 0,006 et 0,014 et sont proches de la valeur optimale 0 témoignant que le modèle est bien ajusté. Ces résultats sont similaires à ceux de Skaggs et al. (2004) qui ont utilisé le modèle Hydrus-2D pour simuler la dynamique de l'eau par le suivi de la teneur en eau et qui ont obtenu des valeurs de RMSE entre 0,001 et 0,04 . Des résultats similaires ont été obtenus par d'autres auteurs (Abbasi et al., 2003a, 2003b; Assouline, 2002; Kandelous et Šimůnek, 2010) qui ont vérifié l'efficacité de Hydrus-2D à simuler la dynamique de l'eau dans le sol (textures variées) avec un système d'irrigation enterrée.

\subsection{Résultats de simulation}

La distribution de l'eau dans le sol autour d'un goutteur enterré dépend principalement de la texture du sol, du débit $\mathrm{du}$ goutteur et des prélèvements racinaires. La variation de la teneur en eau dans le sol à différentes profondeurs (pour des profondeurs d'installation de 15,25 et $35 \mathrm{~cm}$ ) est représentée sur la Figure 4. Les valeurs de la teneur en eau concernant le GGE à $35 \mathrm{~cm}$ de profondeur sont à peu près constantes à proximité de la surface du sol, ce qui est relativement plus stable que pour le GGE à 25 et $15 \mathrm{~cm}$ de profondeur. 


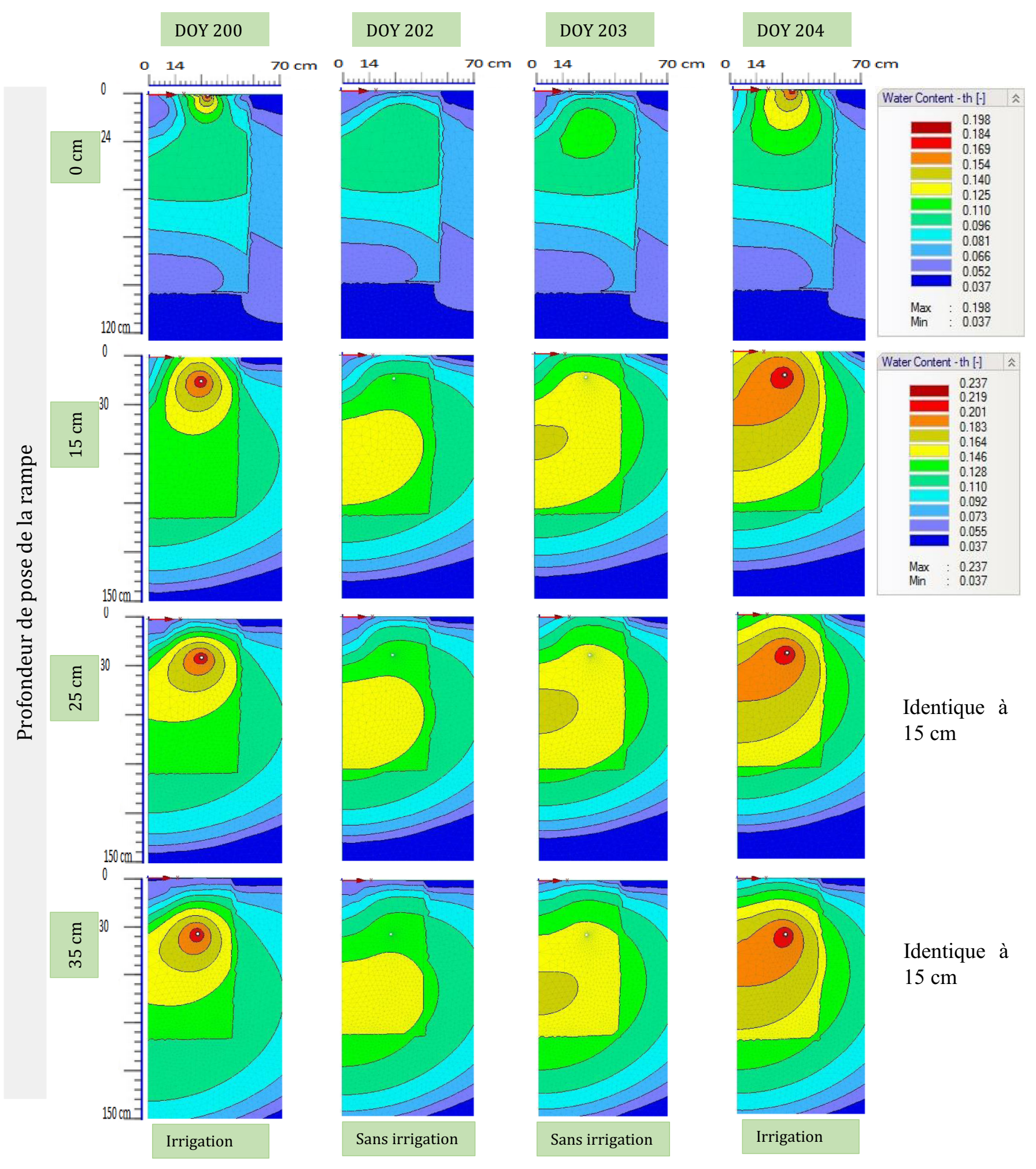

Fig. 4. Simulation de la teneur en eau dans le sol (DOY 200 à 204) avec irrigation et sans irrigation pour les différentes profondeurs du goutte à goutte enterré $(15,25$ et $35 \mathrm{~cm}$ ). L'arbre (positionné à $r=0)$ définit l'axe vertical de symétrie mis en œuvre dans le modèle. Le bulbe est généré par les goutteurs situés à $r=30 \mathrm{~cm}$. Le bulbe vu en coupe occupe un cercle autour de l'arbre (goutteurs proches les uns des autres).

Fig. 4. Simulation of soil water content (DOY 200-204) with irrigation and without irrigation for different depth placement of subsurface drip irrigation $(15,25$ and $35 \mathrm{~cm}$ ). The tree (positioned at $\mathrm{r}=0$ ) defines the vertical axis of symmetry implemented in the model. The wet bulb is generated by the emitters located at $\mathrm{r}=30 \mathrm{~cm}$. The wet bulb seen in section occupies a circle around the tree (emitters close to each other). 
Cependant, la teneur en eau diminue en fonction du temps, en raison de l'augmentation des besoins en eau de la plante.

En été (de juin à août), les précipitations sont rares, de sorte que tous les approvisionnements en eau proviennent de l'eau d'irrigation. Le taux élevé d'évaporation pendant cette période provoque le début de la sénescence observée des feuilles du palmier dattier, en particulier pour les plantations en irrigation GGS et en irrigation GGE à $15 \mathrm{~cm}$ de profondeur. Les profondeurs de 25 et $35 \mathrm{~cm}$ du GGE ont permis d'éviter ce problème de sénescence puisque l'humidité du sol est plus stable et uniforme à $35 \mathrm{~cm}$ de profondeur qu'à 15 et $25 \mathrm{~cm}$. Douh et Boujelben (2012) ont trouvé des résultats similaires dans leur étude comparant le GGS et le GGE pour leur effet sur la variation de la teneur en eau à différentes profondeurs. Ils ont montré que la moyenne de la teneur en eau est plus importante pour le GGE à $35 \mathrm{~cm}$ de profondeur.

Les valeurs simulées de la variation de la teneur en eau $(\Delta \theta)$, en fonction de la profondeur d'enfouissement de la rampe d'irrigation présentent le mouvement latéral de la teneur en eau $\theta=f(r)$ et le mouvement vertical de l'eau $\theta=f(z)$. D'après l'analyse de la Figure 4, nous affirmons que le rayon maximum est toujours au-dessous de l'emplacement du goutteur. Au-delà de ce rayon, qui traduit le mouvement latéral maximum, la variation de la teneur en eau, qui traduit l'avancement du bulbe, est très faible. Ceci est confirmé par Hammami (2001) qui a montré que la forme du bulbe humidifié par un goutteur est plus allongée en profondeur, surtout dans les sols sableux comparés aux sols argileux. Cependant, dans notre cas, il apparaît une discontinuité de la teneur en eau, due à la juxtaposition de matériaux de texture différente. Cette discontinuité explique que pour un même potentiel hydrique nous avons deux humidités différentes à cause de la texture différente entre les deux matériaux.

L'humidité du sol varie en fonction de la profondeur du sol, la distance à l'émetteur et l'emplacement de ce dernier. Selon nos résultats, lorsque la profondeur des goutteurs augmente, la profondeur des mouvements de l'eau augmente, ce qui favorise l'absorption d'eau par les racines. Ce résultat confirme que le système d'irrigation a un effet significatif sur la croissance des feuilles et des racines de palmiers : ceci confirme l'effet observé chez un agriculteur par Bourziza et al. (2014), qui est le plus positif pour les installations en GGE les plus profondes. Selon nos résultats de simulation (Fig. 4), le GGE permet une répartition de l'eau plus verticale qu'horizontale, expliquant ainsi que les racines se développent plus en profondeur que latéralement (Bourziza et al., 2014). Ces résultats sont intéressants puisque les phœniciculteurs pourraient ainsi remédier au problème de l'instabilité des arbres généré par le GGS qui incite les racines à se développer près de la surface du sol, et donc améliorer la résistance du palmier dattier à la sévérité des conditions climatiques des zones oasiennes (vitesse du vent, ensablement), surtout pour les jeunes palmiers dattiers en phase de déploiement de leurs racines.

\section{Conclusion}

L'étude comparative des résultats obtenus par le modèle bidimensionnel axisymétrique Hydrus-2D et les mesures effectuées sur un sol limoneux dans la parcelle d'un agriculteur, en utilisant le GGE installé à différentes profondeurs, a montré la pertinence du modèle pour simuler les processus d'infiltration autour d'une rampe enterrée. Les valeurs de RMSE proche de zéro témoignent de la performance du modèle à simuler correctement la dynamique de l'eau dans le sol. Cependant, quelques limites persistent, telles que l'utilisation de racines statiques pour la simulation, parce que le modèle Hydrus-2D ne permet pas d'entrer la dynamique de croissance racinaire, ce qui permettrait d'illustrer la croissance du système racinaire selon le système d'irrigation.

Les résultats ont également montré que l'humidité du sol est à la fois plus stable et plus importante avec le GGE à $35 \mathrm{~cm}$ de profondeur qu'avec des profondeurs de 15 et $25 \mathrm{~cm}$. En outre, pour une durée égale d'irrigation, l'eau d'irrigation n'atteint pas la surface du sol pour l'installation la plus profonde $(35 \mathrm{~cm})$; c'est le principal avantage du GGE, puisque ce système permet la diminution des pertes par évaporation. La disponibilité de plus d'eau favorise un développement optimum du système racinaire, la croissance de la plante et par la suite une amélioration du rendement et de l'efficience de l'utilisation de l'eau. Par conséquent, le système du GGE permet une économie d'eau, particulièrement appréciable dans les zones où l'eau est une ressource limitée, telles que les oasis.

Remerciements. Cette étude a été réalisée dans le cadre du projet Millenium Challenge Account/Projet arboriculture fruitière «MCA/PAF». Les auteurs remercient M. Sabri et Mme El Khoumsi pour leur aide dans les travaux de terrain.

\section{Références}

Abbasi F, Šimůnek J, Feyen J, van Genuchten MT, Shouse PJ. 2003a. Simultaneous inverse estimation of soil hydraulic and solute transport parameters from transient field experiments: homogeneous soil. Transactions of ASAE 46(4): 1085-1095.

Abbasi F, Jacques D, Šimůnek J, Feyen J, van Genuchten MT. 2003b. Inverse estimation of the soil hydraulic and solute transport parameters from transient field experiments: heterogeneous soil. Transactions of ASAE 46(4): 1097-1111.

Ajdary K, Singh DK, Singh AK, Khanna M. 2005. Simulation of water distribution under drip irrigation using Hydrus-2D. In: XII World water Congress "Water for sustainable developmenttowards innovative solutions", 22-25 November 2005, New Delhi, India, pp. 221-231.

Akbar AK, Yitayew M, Warrick AW. 1996. Field evaluation of water and solute distribution from a point source. J Irrig Drain Eng 122 (4): 221-227.

Assouline S. 2002. The effects of microdrip and conventional drip irrigation on water distribution and uptake. Soil Sci Soc Am J 66: $1630-1636$.

Bourziza R, Hammani A, Kuper M, Bouaziz A. 2014. Économie d'eau en zones arides : comparaison de techniques innovantes pour l'irrigation de jeunes palmiers dattiers. In: Séminaire "Les économies d'eau dans les systèmes irrigués : retours d'expériences au Maroc et à l'international » ANAFIDE/ AFEID, 20-21 octobre 2014, Rabat, Maroc.

Camp CR. 1998. Subsurface drip irrigation: a review. Transactions of the ASAE 41: 1353-1367.

Diamantopoulos E, Elmaloglou S. 2012. The effect of drip line placement on soil water dynamics in the case of surface and subsurface drip irrigation. Irrigation and Drainage 61 (5): 622-630. 
Douh B, Boujelben A. 2012. Subsurface drip irrigation and water management under semiarid climate. Advances in Environmental Research 22: 181-197.

El Khoumsi W, Hammani A, Kuper M, Bouaziz A. 2014. Dynamique des nappes phréatiques dans les milieux arides : quels impacts sur l'écosystème oasien? In: Séminaire «Les économies d'eau dans les systèmes irrigués : retours d'expériences au Maroc et à l'international » ANAFIDE/ AFEID, 20-21 octobre 2014, Rabat, Maroc.

Elmaloglou S, Diamantopoulos E. 2009. Simulation of water dynamics under subsurface drip irrigation from line sources. Agric Water Manage 96: 1587-1595.

Hammami M. 2001. Nouvelle approche pour déterminer le volume de sol humidifié par un goutteur. Thèse de doctorat d'état es-sciences physiques, Faculté des sciences mathématiques, physiques et naturelles de Tunis, Tunisie, $190 \mathrm{p}$.

Hanson B, May D. 2007. The effect of drip line placement on yield and quality of drip irrigated processing tomatoes. Irrig Drainage Syst 21: 109-118.

Hillel D. 1980. Application of soil physics. New York: Academic Press, $385 \mathrm{p}$.

Kandelous MM, Šimůnek J. 2010. Numerical simulations of water movement in a subsurface drip irrigation system under field and laboratory conditions using HYDRUS-2D. Agric Water Manage 97: 1070-1076.

Lazarovitch N, Warrick AW, Furman A, Šimůnek J. 2007. Subsurface water distribution from drip irrigation described by moment analyses. Vadose Zone J 6: 116-123.

Mailhol JC, Ruelle P, Nemeth I. 2001. Impact of fertilization practices on nitrogen leaching under irrigation. Irrigation Science 20: 139-147.

Mailhol JC, Ruelle P, Dejean C, Rosique P. 2013. Le goutte à goutte enterré : une solution innovante pour irriguer sous conditions restrictives en eau. Sciences Eaux \& Territoires 11: 26-29.
Mualem Y. 1976. A new model for predicting the hydraulic conductivity of unsaturated porous media. Water Resour Res 12: 513-522.

Patel N, Rajput TBS. 2008. Dynamics and modeling of soil water under subsurface drip irrigated onion. Agric Water Manage 95 (12): 1335-1349.

Payero JO, Yonts CD, Irmak S, Tarkalson D. 2005. Advantages and disadvantages of subsurface drip irrigation. Extension. EC05-776, Institute of Agriculture and Natural Resources at the University of Nebraska-Lincoln, Nebraska. Available on: http://digitalcommons. unl.edu/extensionhist/4787/.

Provenzano G. 2007. Using HYDRUS-2D simulation model to evaluate wetted soil volume in subsurface drip irrigation systems. J Irrig Drain Eng 133: 342-349.

Rassam L, Littleboy M. 2003. Identifying vertical and lateral components of drainage flux in hillslopes. In: Proceeding of MODSIM 2003. Modeling and Simulation Society of Australia and New Zealand, July 2003, Townsville, Queensland.

Safi B, Neyshabouri MR, Nazem AH. 2007. Water application uniformity of subsurface drip irrigation system at various operating pressures and tape lengths. Turk J Agric Forest 31 (2007): 275-285.

Šimůnek J, Sejna M, Van Genuchten MT. 1999. The HYDRUS-2D software package for simulating the two-dimensional movement of water, heat, and multiple solutes in variably-saturated media. IGWMC-TPS 53, Version 2.0, International Ground Water Modeling Center, Colorado School of Mines, Golden, Colorado, USA.

Skaggs TH, Trout TJ, Šimůnek J, Shouse PJ. 2004. Comparison of HYDRUS-2D simulations of drip irrigation with experimental observations. J Irrig Drain Eng 130 (5): 304-310.

Van Genuchten MT. 1980. A closed-form equation for predicting the hydraulic conductivity of unsaturated soils. Soil Sci Soc Am J 44: 892-898.

Zur B. 1996. Wetted soil volume as design objective in trickle irrigation. Irrig Sci 16: 101-105.

Citation de l'article : Bourziza R, Hammani A, Mailhol JC, Bouaziz A, Kuper M. 2017. Modélisation de l'irrigation en goutte à goutte enterré du palmier dattier sous les conditions oasiennes. Cah. Agric. 26: 35007. 